\title{
Effect of Acetamide Derivative and its Mn- Complex as Corrosion Inhibitor for Mild Steel in Sulphuric Acid
}

\author{
O.S. Shehata*, Lobna A. Khorshed and H. S. Mandour \\ Inorganic, Chemical Industries and Mineral Resources \\ Division, Physical Chemistry Department, National Research \\ Centre, Dokki, Giza, Egypt.
}

\begin{abstract}
H YDRAZONE ligand N-(4-(2-hydrazinyl-2-oxoethoxy) phenyl)- acetamide [HL] and its metal complex of manganese (II) $[\mathrm{Mn}-\mathrm{HL}]$ were investigated as corrosion inhibitors for mild steel in diluted sulphuric acid solution at $25 \pm 1^{\circ} \mathrm{C}$ using three electrochemical methods which show matching results. they are potentiodynamic polarization, cyclic voltammetry \& electrochemical impedance spectroscopy techniques. The experimental results clarify the inhibitive effect of $\mathrm{N}$-(4-(2-hydrazinyl-2-oxoethoxy) phenyl) acetamide [HL] and its Mn (II) complex on mild steel; that increase in presence of the complex [Mn-HL] more than [HL] where The ligand displayed low efficiencies as corrosion inhibitor against localized corrosion while complex gives more corrosion inhibition efficiency. Detailed study effect of $0.1 \mathrm{M}$ sulphuic acid with and without ligand and its complex ligand $\mathrm{MnCl}_{2}$ on mild steel shown by surface morphology of specimen using scanning electron microscope (SEM) and energy dispersive X-ray (EDX) analysis that show improvement appears in surface morphology of mild steel with complex of ligand from localized attack to general attack (homogeneous surface and protective film).
\end{abstract}

Keywords: Acetamide derivative, Sulphuric acid, Mild steel, cyclic voltammetry, EDX.

Recently, according to the growing attention of the world towards environmental problems and towards the protection from hazardous effects of the use of chemicals on ecological balance, the using of tradition al corrosion inhibitors has gradually changed. The researches, therefore, focus on synthetic organic inhibitor and drugs as corrosion inhibitors of steel in acidic solutions. The research performed mainly in the past years identified numerous active drug components that show inhibited effectively on the corrosion of metals and alloys [1-4]. Gece, was identified about 17 classes of drugs that can be used as corrosion inhibitors for $\mathrm{Al}$ and $\mathrm{Al}$ alloys, $\mathrm{Cu}, \mathrm{Zn}$ and $\mathrm{Ti}$ ) in various corrosive environments (solution s of $\mathrm{HCl}, \mathrm{H}_{2} \mathrm{SO}_{4}, \mathrm{H}_{3} \mathrm{PO}_{4}$ and $\mathrm{NaCl}$ ) [5]. Mild steel is strong alloy has good mechanical properties, make it one of the most important engineering materials. It

*Corresponding author E-mail address: omniashehata@yahoo.com

Tel: 002 01150391469Fax.: (+202)33370931C

DOI: $10.21608 /$ ejchem.2017.674.1014

(C2017 The National Information \& Documentation Center(NIDOC) 
was considered the most important structural material exposed to outdoor conditions in acidic media [6]. Acidic solutions are extensively used in acid cleaning, pickling and descaling processes, as well as for drilling operations in oil and gas exploration [7]. Where corrosion is considered more severe due to the general aggressiveness of acid solutions. Inhibitors are commonly used to reduce corrosion resulted from corrosive attack of the metallic materials $[8,9]$. Most organic inhibitors adsorb on the metal surface by displacement water molecules of the surface [10] and forming a compact barrier. Therefore, selection of an appropriate inhibitor from organic compounds [11-13] for acidic environment is of great importance for giving long life to metal. The inhibition process has been shown to occur by inhibitor adsorption, and the efficiency of an inhibitor strongly depends on the structure and their functional groups. A large number of organic compounds containing nitrogen, oxygen, or sulphur have been used as inhibitors to control acid corrosion of iron and steel [14,15]. Several organic compounds such as Acetaminophenol (paracetamol) and its derivative are an active constituent of several pharmaceutical products, extensively used as an analgesic [16,17]. Synergistic effects must be taken into account when drugs contain several active substances. In this paper, we study the inhibiting effect when used a derivative of paracetamol (N-(4- (2-hydrazinyl-2oxoethoxy)phenyl) acetamide) HL and its Mn (II) complex (Mn-HL) on the corrosion of mild steel in $0.1 \mathrm{M}$ sulfuric acid at room temperature.

\section{Materials}

\section{Materials and Experimental}

Material used in this study was type mild steel has chemical analysis as follows (C 0.047\%, Mn 0.289, Si 0.037\%, P 0.017, S 0.013, Cu 0.054, Cr 0.049, Ni 0.057, Ti 0.02, Al 0.003\%, Mo 0.002, Sn 0.02\%) the rest Fe \% and it had active surface $1 \mathrm{~cm}^{2}$. Both inhibitors used N-(4-(2-hydrazinyl-2-oxoethoxy) phenyl) -acetamide (HL) were prepared in the lab and presented as following figures:<smiles>CC(=O)Nc1ccc(OCC(N)=O)cc1</smiles>

Fig.1 a. The structure of ligand (HL).

And its complex [(HL) $\left.\left.2 \mathrm{Mn}_{3} \mathrm{Cl}_{6}\right] 3.5 \mathrm{EtOH} .3 \mathrm{H}_{2} \mathrm{O}\right]$ as [Mn-HL]

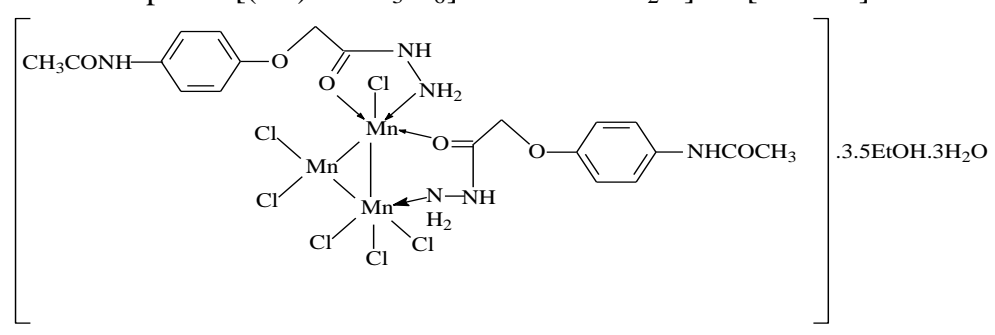

Fig.1 b. the structure of complex of ligand. 
Electrolyte media prepared by dissolved inhibitors with bi-distilled water in the same concentrations $0.004 \mathrm{~g} / \mathrm{l}$ was used throughout all the experiments. Aggressive solution of sulphuric acid was purchased from Al-Gomhoria company, Egypt, of analytical grade $(98.8 \%)$ and prepared with $0.1 \mathrm{M}$ in stock solution of inhibitors.

\section{Experimental}

The electrochemical experiments were carried by using workstation Auto lap $302 \mathrm{~N}$ potentiostate/galvanostate instrument, with using a conventional threeelectrode cell, Working electrode mild steel (WE), platinum counter electrode (CE) and reference electrode (RE) $\mathrm{Ag} / \mathrm{AgCl}$, was immersed in $0.1 \mathrm{M} \mathrm{H}_{2} \mathrm{SO}_{4}$ as aggressive solution with and without inhibitors. Electrochemical impedance spectroscopy (EIS) was probed at the steady open circuit potential using a $10 \mathrm{mV}$ peak-to-peak AC excitation Signal in the frequency domain from $100 \mathrm{KHz}$ to $0.01 \mathrm{~Hz}$. Corrosion current density $\left(\mathrm{i}_{\text {corr }}\right)$ which is equivalent to the corrosion rate of the specimen was estimated using Tafel extrapolation with software NOVA 1.10 from Potentiodynamic Polarization Test at scan rate $2.0 \mathrm{mV} \mathrm{s}^{-1}$. Cyclic voltammetry was studied firstly, on cylinder platinum electrode with dimensions ( 0.25 diammeter, $5 \mathrm{~cm}$ length) as working electrode with electrolytes ligand and its complex at scan rate 1.0 and $2.0 \mathrm{mV} \mathrm{s}^{-1}$, to assessment the ligand and its complex adsorption. And secondly, on mild steel as working electrode in aggressive media sulphuric acid with and without inhibitors to measure the inhibiting efficiency value of them connected to platinum wire as counter electrode (CE) and reference electrode (RE) $\mathrm{Ag} / \mathrm{AgCl}$, at scan rate $1.0 \mathrm{mV} \mathrm{s}^{-1}$. The surface morphology and the percentage of elemental composition of each sample after immersion were carried out using by Field Emission Gun (F.E.G.) Model Quanta 250 to compare the surface morphologies of specimens after various surface treatments.

\section{Results}

Open Circuit Potential measurements (OCP)

Figure 2 represented the open circuit potentials of mild steel versus time was recorded for aggressive media $0.1 \mathrm{M}$ sulphuric acid without and with ligand (HL) and its complex $(\mathrm{Mn}-\mathrm{HL})$ in $4 \times 10^{-3} \mathrm{~g} / \mathrm{L}$ over period $600 \mathrm{~s}$. The corrosion potential all have similar trend increases until the passive film reaches its limiting protective capacity, resulting in stabilization of the corrosion potential, the electrochemical reactions at mild steel-electrolyte interface vary with time. It is found that the potential starts to drift slowly to the more negative direction with $\mathrm{Mn}-\mathrm{HL}-0.592 \mathrm{mV}$, while increase in less negative direction with (HL) till about $500 \mathrm{~s}$ then reach the maximum stabilization potential at $-0.525 \mathrm{mV}$ for $\mathrm{HL}$ which above $0.1 \mathrm{M}$ sulphuric acid at $-0.530 \mathrm{mV}$. 


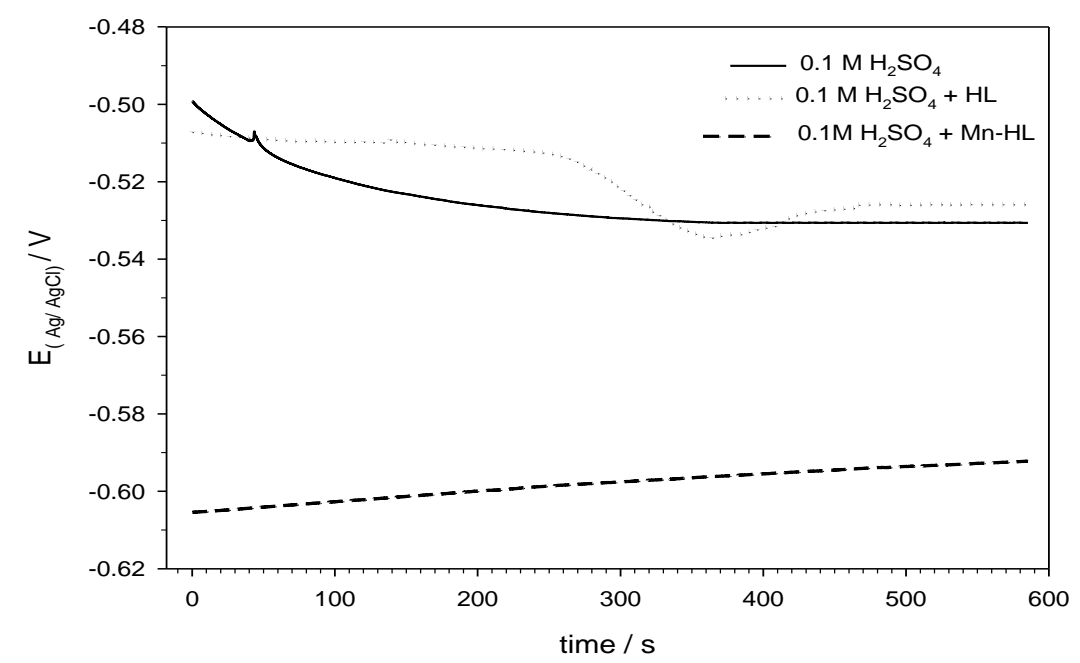

Fig. 2. Time dependence of the Open Circuit Potential (OCP) for mild steel in $0.1 \mathrm{M}$ $\mathrm{H}_{2} \mathrm{SO}_{4}$ with and without inhibitors at $25^{\circ} \mathrm{C}$.

Electrochemical impedance spectroscopy

Electrochemical impedance spectroscopy is extremely effective tool in the study of surface properties of metal, and gives information about inhibitor adsorption, EIS measurement occurs at room temperature. Figure 3a shows Bode plots and Fig. 3b Nyquist obtained from impedance data. The impedance spectra in Bode show resistive region between high (HF) and low (LF) frequencies, where the spectra shows increase impedance value over LF range with inhibited solution of $0.1 \mathrm{M} \mathrm{H}_{2} \mathrm{SO}_{4}$ with $\mathrm{HL}$ and $\mathrm{Mn}-\mathrm{HL}$ this be associated with increase in phase maximum from 40 in sulphuric acid without inhibitor to 50 showing higher shift in phase towards higher frequency in case of inhibited media as indication to higher stability for film and faster reaction of adsorption forming protective film its interfacial thickness according to Helmholtz equation [17] is relative thickness $(\delta)$ proportional to $C$ as equation (1):

$$
C=\varepsilon \varepsilon_{o} A / \delta
$$

where $\varepsilon_{0}$ is the vacuum dielectric constant, $\varepsilon$ local dielectric constant of metal, $\delta$ is thickness of the interfacial layer and $A$ is the surface area of the electrode as shown in Fig. 4. The decrease in $C$ might be to replace the adsorbed (water high dielectric constant) by the ligand and its complex (low dielectric constant) adsorbed on mild steel surface, The capacitance is inversely proportional to the thickness of the interfacial layer and increase charge-transfer resistance. It is clear that the values of capacitance decrease in the presence of inhibitors due to the increase of interfacial layer at the metal solution interface and the regular replacement of water molecules according to the adsorption of inhibitors on the metal surface [18].

Egypt. J. Chem. 60, No. 2 (2017) 

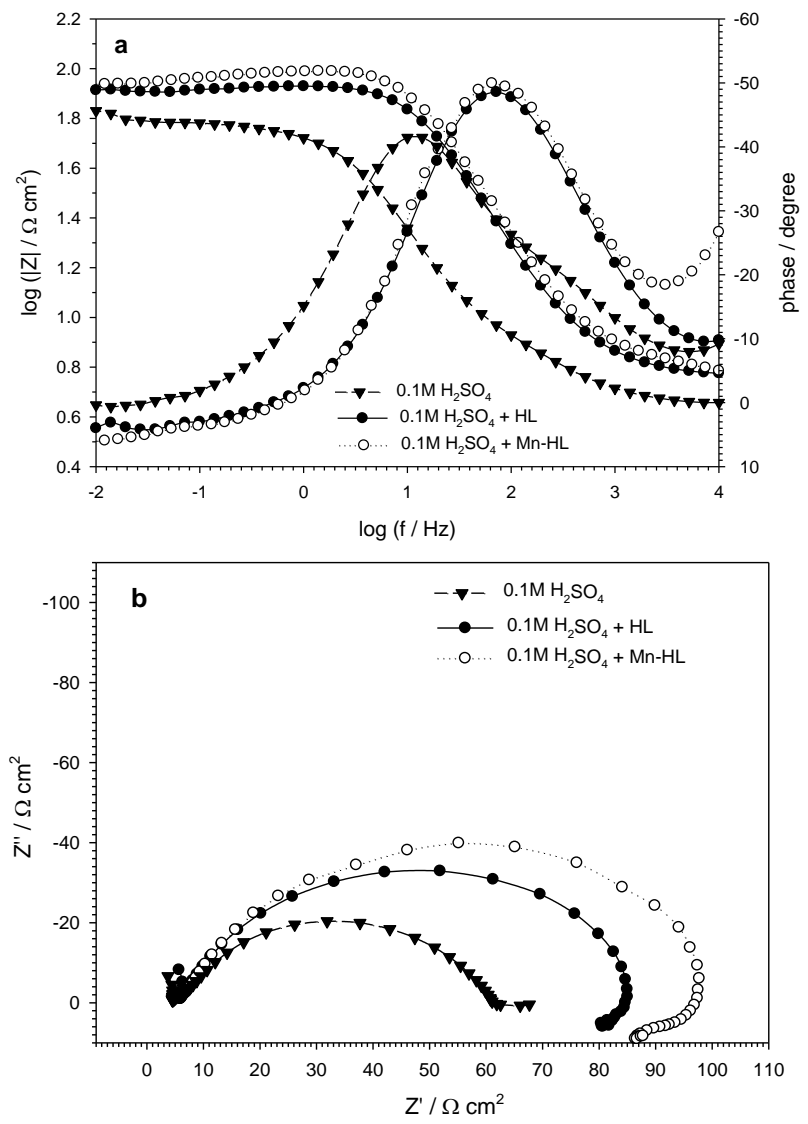

Fig. 3. Electrochemical impedance spectra for mild steel in $0.1 \mathrm{M} \mathrm{H}_{2} \mathrm{SO}_{4}$ with and without inhibitors as (a) Bode plot; and (b) Nyquist plot at $25^{\circ} \mathrm{C}$.

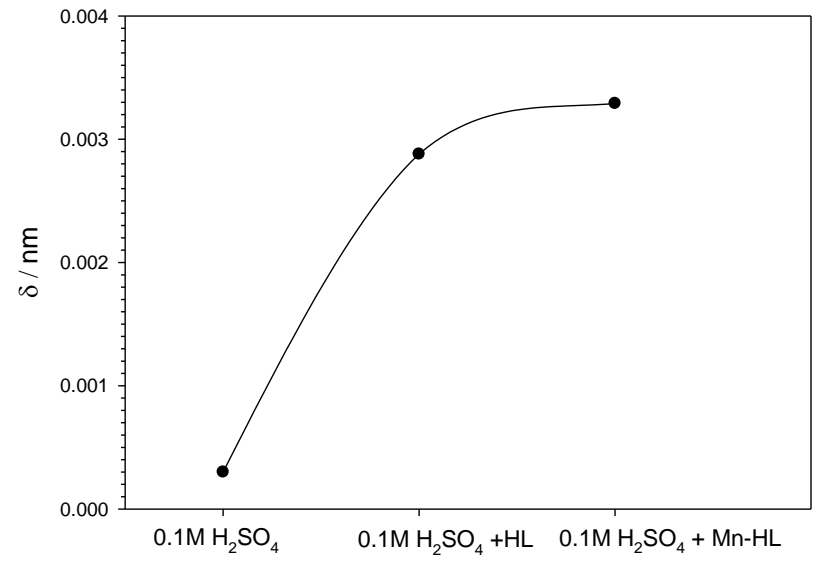

Fig. 4. Dependence thickness film on mild steel in $0.1 \mathrm{M} \mathrm{H}_{2} \mathrm{SO}_{4}$ with and without inhibitors at $25^{\circ} \mathrm{C}$. 
The Nyquist plot show single depressed semicircles whose diameters increased with presence inhibitors in case of $\mathrm{HL}$ than $0.1 \mathrm{M} \mathrm{H} \mathrm{H}_{2} \mathrm{SO}_{4}$ while $(\mathrm{Mn}$ $\mathrm{HL}$ ) give the greatest diameter which evidences the powerful effect of both ligand and its derivatives on adsorption efficiency. This indicates that the presence of ligand and its complex increase corrosion resistance. This was confirmed with fitting the EIS data to simplified Randle equivalent, as shown in Fig. 5.

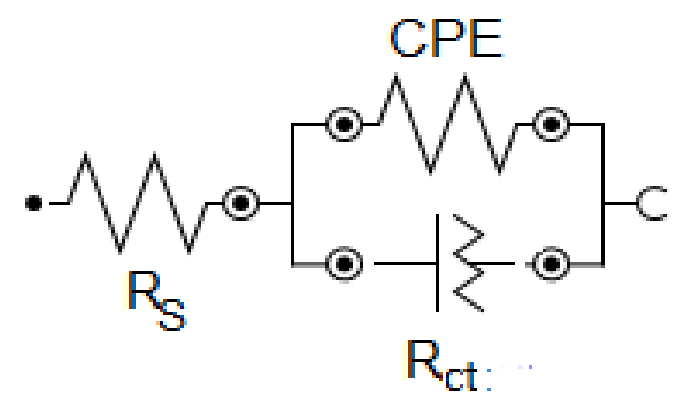

Fig. 5. The electrochemical equivalent circuit used to fit the impedance data.

The three parameters of circuit can be defined as order: $(R S)$ is solution resistance $(C P E)$ is constant phase elements, $R_{\mathrm{ct}}$ is charge transfer resistance of solution / steel interface. Fitting data from using equivalent circuit parameter are listed in Table 1 . We noticed that Charge transfer resistance $R_{c t}$ increase in presence of ligand and its complex than its value in sulphuric acid this increases be associated with a lower corrosion rate and presence more protective film on the surface of mild steel whereas presence complex (Mn-HL) give the most effective, values of $C P E$ decrease in inhibited media than uninhibited giving the lowest value with complex (Mn-HL) which would result from a decrease in dielectric constant and might increase in thickness of protective film this approved adsorption at the metal solution interface that show with increasing values of $\alpha$ from 0.685 to 0.843 and 0.856 with both inhibited cases.

TABLE 1. Electrochemical impedance parameters for mild steel in $0.1 \mathrm{M} \mathrm{H}_{2} \mathrm{SO}_{4}$ solution in the absence and presence ligand (HL) and its complex (MnHL) at room temperature.

\begin{tabular}{lcccc}
\hline Corrosive media & $\begin{array}{c}R_{C T} \\
\Omega . \mathrm{cm}^{2}\end{array}$ & $\begin{array}{c}C P E \\
\mu \mathrm{F} / \mathrm{Cm}^{2}\end{array}$ & $A$ & $\begin{array}{c}R_{s} \\
\Omega . \mathrm{cm}^{2}\end{array}$ \\
\hline $0.1 \mathrm{M} \mathrm{H}_{2} \mathrm{SO}_{4}$ & 41.9 & 3350 & 0.685 & 3.10 \\
$0.1 \mathrm{M} \mathrm{H}_{2} \mathrm{SO}_{4}+\mathrm{HL}$ & 54.6 & 347 & 0.843 & 4.13 \\
$0.1 \mathrm{M} \mathrm{H}_{2} \mathrm{SO}_{4}+\mathrm{Mn}-\mathrm{HL}$ & 61.1 & 304 & 0.856 & 4.24 \\
\hline
\end{tabular}

The synergistic effect of complex attributed to large number of its constituents; nitrogen, oxygen and manganese (II) atoms or structures containing $\pi$-electrons in their molecules [19-21] in Mn-HL complex which show more resistance has 
molecular weight 1039.107 than that of ligand itself which has molecular weight 223.23 this is may be attributed to the structure of the complex [22].

\section{Potentiodynamic Polarization test}

Mild steel in $0.1 \mathrm{H}_{2} \mathrm{SO}_{4}$ with and without ligand and complex are study by potentiodynamic polarization method at scan rate $2 \mathrm{mV} / \mathrm{s}$. Results show in Fig. 6 that current density in the anodic region increases for mild steel in the absence of ligand and its complex rapidly indicating extensive dissolution of metal. This led to both the anodic metal dissolution and cathodic hydrogen evolution reactions were controlled after the addition of ligand and its complex to aggressive media which give obvious shifted decrease in cathodic and anodic currents depend on presence ligand and its complex by limiting oxygen diffusion to surface of mild steel, this indicates that presence ligand and its complex impede the attack on mild steel due to forming protective film by adsorption HL and Mn-HL on metal surface.

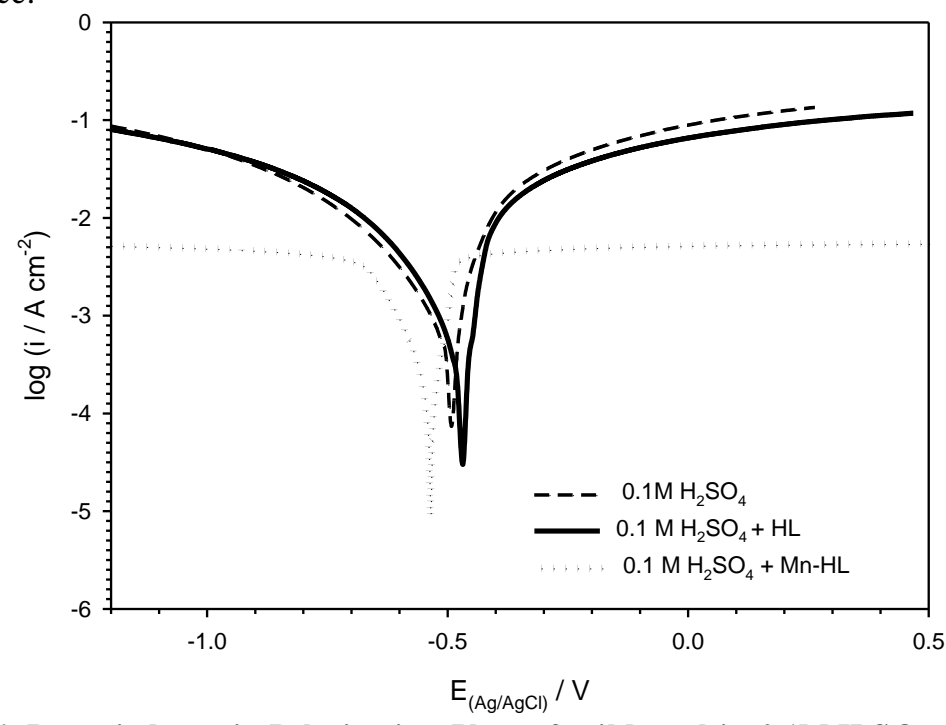

Fig. 6. Potentiodynamic Polarization Plots of mild steel in $0.1 \mathrm{M} \mathrm{H}_{2} \mathrm{SO}_{4}$ with and without inhibitors at scan rate $2 \mathrm{mV} / \mathrm{s}$ at $25^{\circ} \mathrm{C}$.

The electrochemical parameters such as corrosion potential $\left(E_{c o r r}\right)$, corrosion current $\left(I_{c o r r}\right)$, anodic and cathodic Tafel slopes $(\beta a$ and $\beta c)$, that were given according the Stern-Geary equation [23] resistance polarization $R_{p}$ calculated from equation (2), also corrosion rate in $(\mathrm{mm} / \mathrm{y})$ from Faraday's law [24] as equation (3):

$$
\begin{aligned}
& I_{\text {corr }}=1 / R_{p} x \beta a \beta c / 2.303(\beta a+\beta c) \\
& C R=\left(I_{\text {corr }} K . E W\right) / d A
\end{aligned}
$$

where $I_{\text {corr }}$ is the corrosion current in amperes, $K$ is a constant, defined the units for the corrosion rate equal 3272 for $\mathrm{mm} / \mathrm{y}, E W$ is the equivalent weight in grams/equivalent, $d$ density in $\mathrm{g} / \mathrm{cm}^{3}, A$ sample area in $\mathrm{cm}^{2}$, and the inhibition 
efficiency $(\eta \%)$ values at $0.1 \mathrm{M} \mathrm{H}_{2} \mathrm{SO}_{4}$ are recorded in Table 2 . The $\eta \%$ was calculated from polarization measurements according to the relation [25] equation (4):

$$
\eta \%=\left(\mathrm{I}_{\mathrm{corr}}-\mathrm{I}_{\text {corr }}^{\prime} / \mathrm{I}_{\mathrm{corr}}\right) \times 100
$$

where $I_{\text {corr }}$ and $I_{\text {corr }}^{\prime}$ are corrosion current densities in the absence and presence of the inhibitor, respectively.

Table 2 shows that there is irregular displacement in the corrosion potential $\left(E_{c o r r}\right)$ values due to decreasing of corrosion current, Also, the values of $\beta a$ and $\beta c$ are decreased with adding ligand and its complex to aggressive media is evident for presence ligand and its complex act as mixed inhibitors owing to $\mathrm{Li}$ and others [26].

TABLE 2. Electrochemical polarization parameters and the corresponding inhibition efficiencies for mild steel, in $0.1 \mathrm{M} \mathrm{H}_{2} \mathrm{SO}_{4}$ solution in the absence and presence ligand (HL) and its complex (Mn-HL) at $25{ }^{\circ} \mathrm{C}$.

\begin{tabular}{|c|c|c|c|c|c|c|c|}
\hline $\begin{array}{l}\text { Types of } \\
\text { medium }\end{array}$ & $\begin{array}{l}i_{\text {corr }} \\
\mathbf{m A} \\
\mathbf{c m}^{-2}\end{array}$ & $\begin{array}{c}E_{\text {corr }} \\
\mathbf{m V}\end{array}$ & $\begin{array}{l}\beta_{C} \\
\mathbf{m V} \\
/ \text { dec }\end{array}$ & $\begin{array}{l}\boldsymbol{\beta}_{a} \\
\mathbf{m V} \\
/ \mathrm{dec}\end{array}$ & $\begin{array}{l}\text { Corr. rate } \\
\mathrm{mm} / \mathrm{year}\end{array}$ & $\begin{array}{c}R_{p} \\
\Omega . \\
\mathbf{c m}^{2}\end{array}$ & $\eta \%$ \\
\hline $\begin{array}{l}0.1 \mathrm{M} \\
\mathrm{H}_{2} \mathrm{SO}_{4} \\
\end{array}$ & 1.388 & -499.8 & 99.59 & 251.60 & 16.124 & 15.51 & ------- \\
\hline $\begin{array}{l}0.1 \mathrm{M} \\
\mathrm{H}_{2} \mathrm{SO}_{4} \\
+\mathrm{HL}\end{array}$ & 0.792 & $\begin{array}{c}- \\
467.18\end{array}$ & 64.86 & 179.40 & 9.204 & 18.14 & 42.94 \\
\hline $\begin{array}{l}0.1 \mathrm{M} \\
\mathrm{H}_{2} \mathrm{SO}_{4} \\
+\mathrm{Mn}-\mathrm{HL}\end{array}$ & 0.352 & 530.35 & 61.33 & 138.73 & 4.092 & 36.42 & 74.64 \\
\hline
\end{tabular}

So, the results indicate that $\mathrm{HL}$ and $\mathrm{Mn}-\mathrm{HL}$ act as the mixed type corrosion inhibitors where adsorbed ligand and its complex works by simple blocking of active sites for anodic and cathodic processes. These results confirmed with Electrochemical impedance spectroscopy results as shown in Fig. 7 represented how corrosion rate is inversely proportional with charge transfer resistance of mild steel in sulphuric acid as aggressive media with and without inhibitor.

Adsorption is the binding of molecules to the electrode, oxidizing and reducing potentials of the ligand and its complex studied on platinum electrode due to its good electrochemical inertness in chemical reaction by Cyclic voltammetry that considered a simple, rapid, and powerful method for allowing to probe the mechanics of redox and transport properties of a inhibitor in solution. Cyclic voltammetry technique show as relation between potential and current value measured at each value of potential with two different scan rates.

Firstly, Platinum electrode used as working electrode due to its surface not changed by electrochemical reactions taking place by cyclic voltammetry technique, so, the results of electron transfer between the electrode surface and a

Egypt. J. Chem. 60, No. 2 (2017) 
species in solution to be useful analytically, the current potential plots should reflect changes which occur in the concentrations of species in solution and not in the nature of the electrode surface.

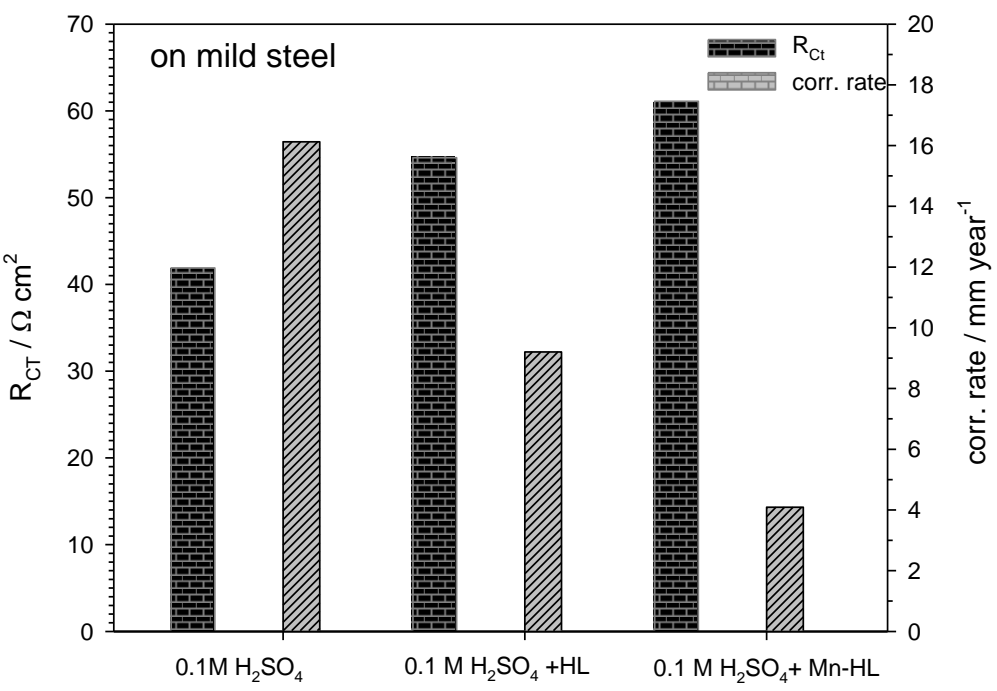

Fig. 7. Charge transfer Resistance and corrosion rate Dependence on mild steel in $0.1 \mathrm{M} \mathrm{H}_{2} \mathrm{SO}_{4}$ with and without inhibitors at $25^{\circ} \mathrm{C}$.

This shown in Fig. 8, the initial scan starting with $-1.0 \mathrm{~V}$ and ending at $1.0 \mathrm{~V}$ plateau show increases in current gradually with changing potential at scan rate $1 \mathrm{mV} / \mathrm{s}$ in Fig. 8a, it is reached $1.99 \mu \mathrm{A}$ in case of $\mathrm{Hl}$ and 0.9 in case Mn-HL with scan rate $1 \mathrm{mV} / \mathrm{s}$ show decreasing in electro oxidation process, while at scan rate $2 \mathrm{mV} / \mathrm{s}$ in fig. 8b extent peaks appear as follows: peak (b) occurs at $-0.6 \mathrm{~V}$ for $\mathrm{Mn}-\mathrm{HL}$ compare to $-0.3 \mathrm{~V}$ for HL to oxygen reduction, and peak (c)occurs at $0.8 \mathrm{~V}$ for $\mathrm{HL}$ compare to $-0.5 \mathrm{~V}$ for $\mathrm{Mn}-\mathrm{HL}$ is attributed to the oxidation of adsorbed hydrogen, while branch (d) to the hydrogen evolution. Maximum peak increases in current reaches $-0.1 \mu \mathrm{A}$ in $\mathrm{HL}$ and $0.1 \mathrm{in} \mathrm{Mn}-\mathrm{HL}$ at the same ending potential range $1.0 \mathrm{~V}$ potential.

Figure 9 shows cyclic voltammetry on mild steel in $0.1 \mathrm{M} \mathrm{H}_{2} \mathrm{SO}_{4}$ in absence and presence of inhibitors (HL) and its complex with scan rate $1 \mathrm{mV} / \mathrm{s}$ that show percentage of the passive film increase as order $0.1 \mathrm{M} \mathrm{H}_{2} \mathrm{SO}_{4}<0.1 \mathrm{M} \mathrm{H}_{2} \mathrm{SO}_{4}+$ $\mathrm{HL}<0.1 \mathrm{M} \mathrm{H}_{2} \mathrm{SO}_{4}+\mathrm{Mn}-\mathrm{HL}$ where peak goes downward in presence both inhibitors than in $0.1 \mathrm{M} \mathrm{H}_{2} \mathrm{SO}_{4}$ as agree with results of others techniques. 


\subsection{Cyclic Voltammetry}
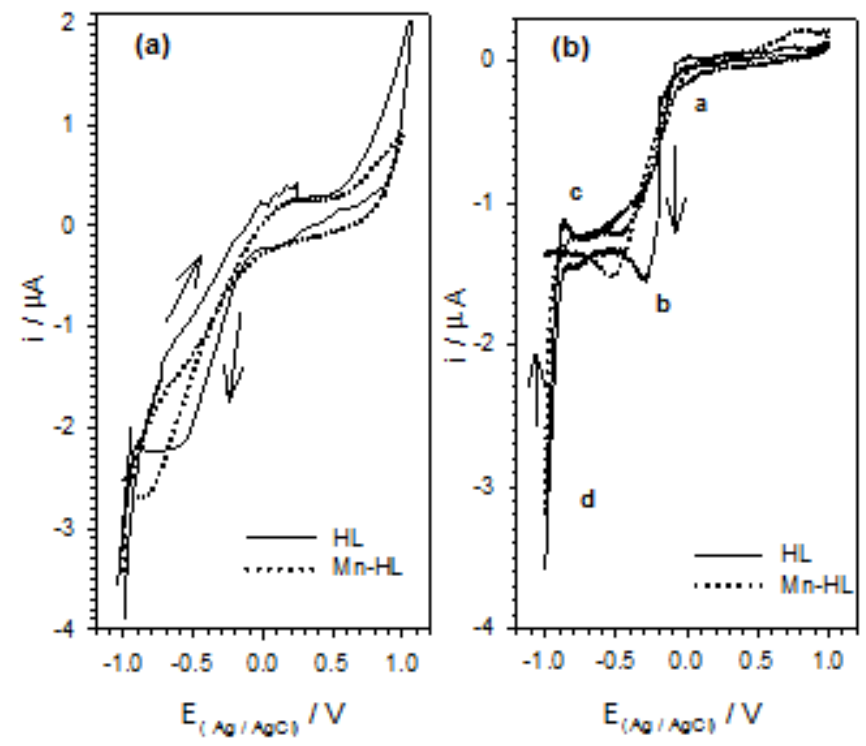

Fig. 8. cyclic voltammetry of Pt electrode in ligand and its complex at different scan rates as follow (a) at scan rate $1 \mathrm{mv} / \mathrm{s}(\mathrm{b})$ at scan rate $2 \mathrm{mv} / \mathrm{s}$ at $25^{\circ} \mathrm{C}$.

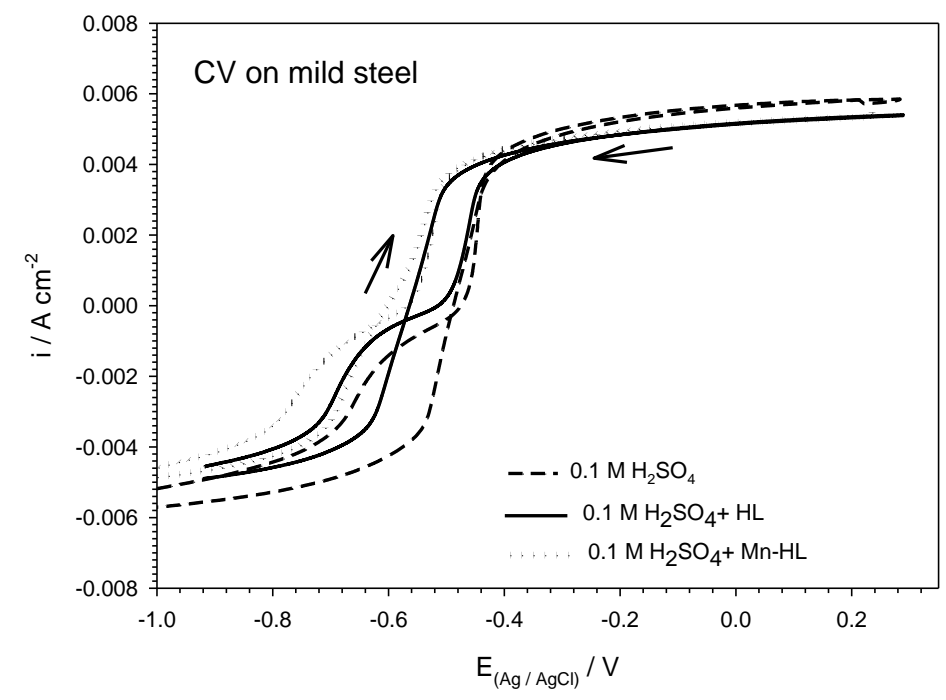

Fig. 9. cyclic voltammetry of mild steel electrode in $0.1 \mathrm{M} \mathrm{H}_{2} \mathrm{SO}_{4}$ with and without inhibitors at scan rate $1 \mathrm{mV} / \mathrm{s}$ at $25^{\circ} \mathrm{C}$.

Egypt. J. Chem. 60, No. 2 (2017) 


\section{Surface examination using SEM-EDX techniques}

SEM and EDX experiments were carried out in order to verify the elements present on the surface mild steel after immersion for 6 hours in $0.1 \mathrm{M}$ sulphuric with and without inhibitors, as shown in Fig.10. from this figure it obvious that there is an improvement in the surface morphology comparing with sample immersed in $0.1 \mathrm{M}$ sulphuric acid without inhibitor, which show in Fig 10 (a) alloy surface suffered from localized corrosion with obvious pitting developed in diluted sulfuric acid after relatively long immersion times (6 hr.), and which was due to the aggressive attack of the sulfuric acid solution.

In contrast in aggressive media with inhibitor, as shown in Fig.10 (b) in presence HL, metallic surface seems to be in most very fine but non homogenous while In case Fig. 10 (c) in presence Mn-HL complex the alloy surface is covered by protecting film and hence a more homogeneous surface is formed due to its more synergistic inhibitive effect. The results of elemental analysis of the surface corrosion products showed the elemental compositions were some variations in the concentration of the elements detect for each shade of the corrosion products in presence and absence inhibitors this attributed to inhibited effect of HL and Mn-HL complex.

\section{Discussion}

Mechanism of mild steel corrosion in $0.1 \mathrm{MH}_{2} \mathrm{SO}_{4}$ without and with inhibitor

Dissolution of mild steel metal is spontaneous process in presence of diluted sulfuric acid, at the anodic areas:

$$
\mathrm{Fe} \rightarrow \mathrm{Fe}^{2+}+2 \mathrm{e}^{-}
$$

There are several different cathodic reactions that are the most common reactionsin acidic media:

$$
\begin{aligned}
& 2 \mathrm{H}^{+}+2 \mathrm{e}^{-} \rightarrow \mathrm{H}_{2} \\
& \mathrm{O}_{2}+4 \mathrm{H}^{+}+4 \mathrm{e}^{-} \rightarrow 2 \mathrm{H}_{2} \mathrm{O}
\end{aligned}
$$



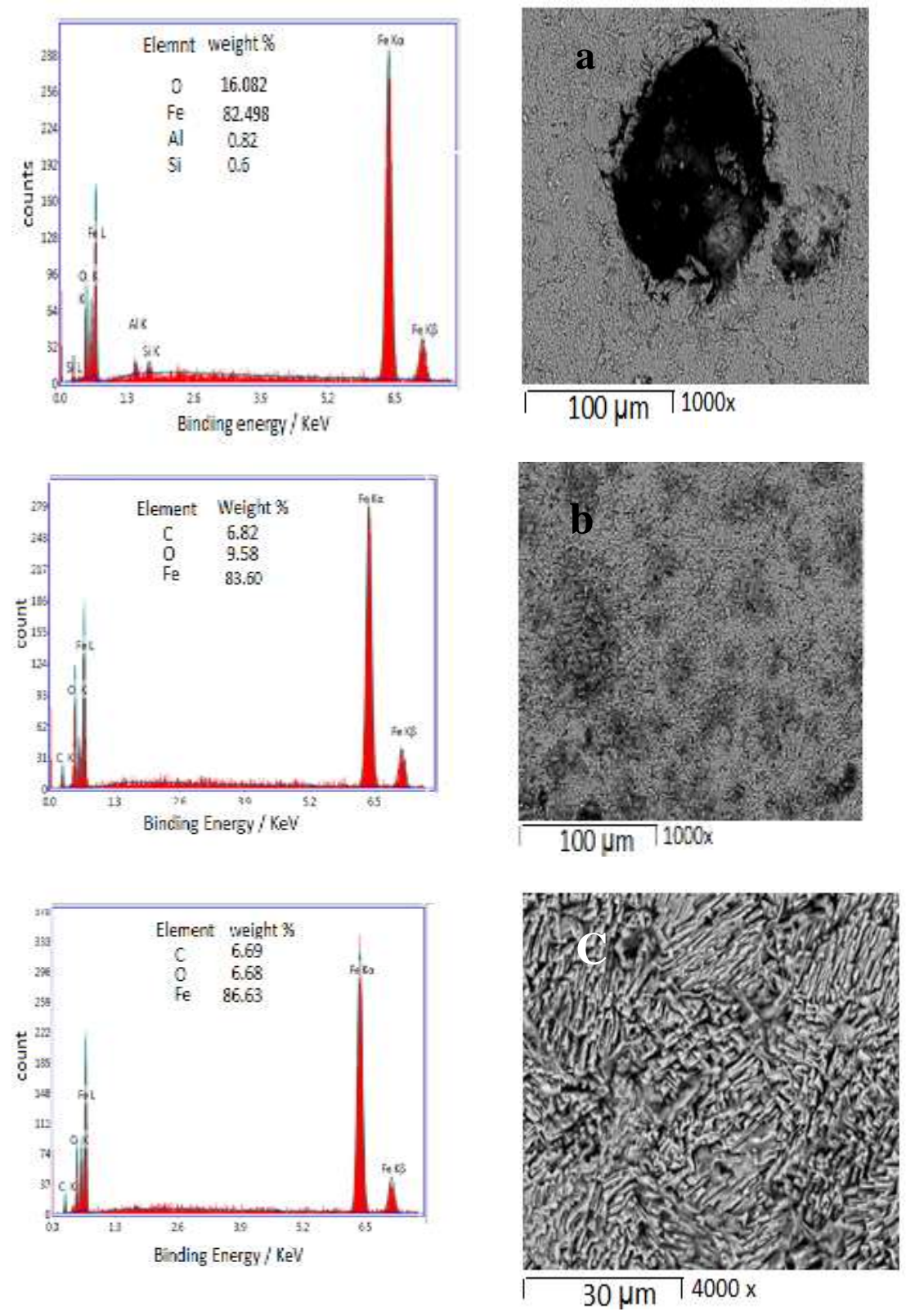

Fig. 10. SEM images and EDX spectra of mild steel in $0.1 \mathrm{M} \mathrm{H}_{2} \mathrm{SO}_{4}$ with and without inhibitors at as follow (a) for $0.1 \mathrm{M} \mathrm{H}_{2} \mathrm{SO}_{4}$ (b) for $0.1 \mathrm{M} \mathrm{H}_{2} \mathrm{SO}_{4}+\mathrm{HL}$ (C) for $0.1 \mathrm{M} \mathrm{H}_{2} \mathrm{SO}_{4}+\mathrm{Mn}-\mathrm{HL}$ at $25^{\circ} \mathrm{C}$.

Egypt. J. Chem. 60, No. 2 (2017) 
When iron corrodes, the rate is usually controlled by the cathodic reaction.

$$
\mathrm{Fe}+\mathrm{H}_{2} \mathrm{SO}_{4} \longrightarrow \mathrm{FeSO}_{4}+\mathrm{H}_{2}
$$

$\mathrm{Fe}$ is oxidised to $\mathrm{Fe}^{2+}$ by $\mathrm{H}^{+}$; the $\mathrm{SO}_{4}{ }^{2-}$ ions was not strong enough to act as an oxidising agent so, it plays no role in the reaction according to Le Chatelier's principle. Reduction reaction is hydrogen gas evolution as follows:

$$
2 \mathrm{H}^{+}+2 \mathrm{e} \longrightarrow \mathrm{H}_{\mathrm{ads}} \longrightarrow 2 \mathrm{H}_{2}
$$

Where $\mathrm{H}_{\mathrm{ads}}$ is hydrogen ions adsorbed on mild steel surface which catalyze to react with another hydrogen ions to contain covert hydrogen gas in bubble form on cathode surface. The amount of hydrogen gas evolved in presence of inhibitor depends on its ability to prevent this reaction and protected the mild steel from corrosion [27].

The action of inhibitor molecules is due to the adsorption of the inhibitor molecules on an exposed mild steel surface. Inhibitors may be adsorbed over the mild steel surface in the form of neutral molecules involving replacement of hydrogen ions adsorbed from the mild steel surface as:

$$
\text { inhibitor }+\mathrm{n} \mathrm{H}_{\mathrm{ads}} \longrightarrow \text { inhibitor }_{\mathrm{ads}}+\mathrm{H}_{2}(10)
$$

or by displacement water molecules of the surface as equation (7)

Organic molecules maybe adsorbed on the mild steel surface in four ways namely; (i) electrostatic interaction between the charged molecules and the charged metal, (ii) interaction of unshared electron pairs in the molecule with the metal, (iii) interaction of $\pi$-electrons with the metal and (iv) a combination of types (i-iii) [28]

In this case of ligand and its complex, the interaction may take place through the interaction of unshared pair of electrons of the nitrogen atom with the alloy surface since the iron surface is positively charged in sulphuric acid medium. The inhibitive properties of amines are mainly dependent on the electron densities around the nitrogen atoms; the higher the electron densities at the nitrogen atom as shown in ligand comparing to its complex, Due to adsorption, inhibitor molecules block the reaction sites and reduce the rate of corrosion reaction. The adsorbed bond strength is determined by the electron density of these atoms $[29,30]$. The inhibition action of $\mathrm{N}$-containing compounds is explained in view of the electron donation of the $\mathrm{N}$ atom through which the molecule could be adsorbed on mild steel (M) as: $\left(\mathrm{M}: \mathrm{NH}_{2} \mathrm{R}\right)$, or by bridging with the active hydrogen atom attached to the $\mathrm{N}$ atoms, as: (N: HNHR). The inhibition efficiency of these compounds was found to depend upon both the electron density on the $\mathrm{N}$ atom and the geometry of the molecule and the chain length. Adsorption of inhibitors usually involves at least two types of adsorption simultaneously, first establishing a physisorbed on crystalline surface film, 
which may further stabilized through chemisorption to form a donor type bond. Chemisorption involves charge charring or charge transfer from the inhibitor molecules to the surface in order to form a coordinate bond [31] .So, we can considered these inhibitors affect corrosion inhibition by their physical and chemical interaction with the alloy surface, forming a protective film, acting as a barrier and increases the activation energy for metal dissolution.

Theoretical explanation of inhibitor function are in common agreement that adsorption mixed molecules phenomena where these are organic molecules with more than one orienting group attached (i.e.; $\mathrm{NH}_{2}$ ) has ability to effect on both anodic and cathodic processes of corrosion. These structures are classified as "ambiodic inhibitors". Where their effective is electron density distribution causes inhibitor to be attracted to both anodic and cathodic areas. The inhibitive action suggested by Mann [32] that organic substance forming onium ions in acidic solutions are adsorbed on the cathodic reaction. We concluded that numerous $\mathrm{OH}$ groups around the molecules make them more ready to form strong links with hydrogen. In addition, they can form complexes with metal Mn (II) which is rarely soluble in aqueous environment. This complex can cause blocking of micro-anodes and/or micro-cathodes that are generated on the mild steel surfaces in contact with electrolytes and so can retard the subsequent dissolution of the mild steel and should fortify ligand through synergism due to it adsorbed on the mild steel surface in combination of electrostatic interaction between the charged molecules and the charged mild steel and interaction of $\pi$ electrons with the mild steel.

\section{Conclusion}

The result obtained in the present study showed that the inhibitor material under investigation ligand and its complex have appreciable inhibitive property. The ligand displayed low efficiencies as corrosion inhibitors against localized corrosion while complex give more efficiency corrosion inhibition this must be attributed to its large number of constituents. It is most probable that this large number of constituents includes anodic, cathodic and general inhibitor molecule. The synergistic action of the compounds could be also noticed from the low concentrations of inhibitor required to attain maximum inhibition. Polarization measurements indicate both can act as a mixed type inhibitor affecting both the cathodic and anodic corrosion reactions. The efficiency of ligand and its complex obtained show good agreement with results from AC impedance results. Cyclic voltammetry study indicates that the addition of inhibitor reduces the current where peak goes downward in presence both ligand and its complex than in $0.1 \mathrm{M} \mathrm{H}_{2} \mathrm{SO}_{4}$. SEM shows improvement appears in surface morphology of mild steel with complex of ligand from localized attack to general attack (homogeneous surface and protective film). 


\section{References}

1. Abdallah, M., Antibacterial drugs as corrosion inhibitors for corrosion of aluminum in hydrochloric solution. Corros. Sci. 46, 1981-1996 (2004).

2. Arslan, T., Kandemirli, F., Ebenso, E.E., Love I. and Alemu, H., Quantum chemical studies on the corrosion inhibition of some sulphonamides. Corros. Sci., 51, 35-47 (2009).

3. Tamborin, S. M., Dias, S. L. P., Silva, S. N., Dick, L. F. P. and Azambuja, D. S., Preparation and electrochemical characterization of amoxicillin-doped cellulose acetate films for AA2024-T3 aluminum alloy coatings. Corros. Sci. 53, 1571-1580 (2011).

4. Xuehui, P., Xiangbin R., FeiK., Jiandong, X. and Baorong, H., Inhibiting effect of ciprofloxacin, norfloxacin and ofloxacin on corrosion of mild steel in hydrochloric acid. Chin. J. Chem. Eng. 18, 337-345 (2010).

5. Gece, G. Drugs: a review of promising novel corrosion inhibitors. Corros. Sci. 53, 3873-3898 (2011)

6. Fekry, A. M. and Mohamed R.R., "Acetyl thiourea chitosan as aneco-friendly inhibitor for mild steel in sulphuric acid medium. Electrochimica Acta, 55 (6), 19331939 (2010),

7. Mahdavian, M., and Ashhari, S., Corrosion inhibition performance of mercaptobenzimidazole and 2-mercaptobenzoxazole compounds forprotection of mild steel in hydrochloric acid solution. Electrochim Acta, 55, 1720-1724 (2010).

8. Wang, H.-L., Liu R.-B. and Xin, J. Inhibiting effects of some mercapto-triazole derivatives on the corrosion of mild steel in 1.0,MHC1medium. Corrosion Science, 46 (10), 2455-2466 (2004).

9. EmregülK, C., Kurtaran, R., and Atakol, O., An investigation of chloridesubstituted Schiff bases as corrosion inhibitors for steel. Corrosion Science, 45(12), 2803-2817(2003).

10.Oguzie, E. E, Li Y and Wang F. H. Corrosion inhibition and adsorption behavior of methionine on mild steel in sulfuric acid and synergistic effect of iodide ion. Journal of Colloid and Interface Science. 310 (1), $90-98$ (2007).

11.Choi, D. J., Kim Y. W. and Kim, J. G. Development of a blended corrosion, scale, and microorganism inhibitor for open recirculating cooling systems. Werkstoffe und Korrosion, 52 (9), 697-704 (2001).

12.Moretti, G., Guidi, F. and Grion, G., Tryptamine as a green iron corrosion inhibitor in $0.5 \mathrm{M}$ deaerated sulphuric acid. Corrosion Science. 46 (2), 387-403 (2004).

13.Abd El-Maksoud, S. A. The effect of organic compounds on the electrochemical behaviour of steel in acidic media-a review. International Journal of Electrochemical Science. 3, 528-555 (2008). 
14.Abdallah, M., Al-Agez, M. and Fouda, A. S. Phenyl hy drazone derivatives as corrosion inhibitors for brass in hydrochloric acid solutions. International Journal of Electrochemical Science. 4 (3), 336-352 (2009).

15.Bouklah, M., Benchat, N. and Aouniti, A. et al., Effect of the substitution of an oxy gen atom by sulphur in a pyridazinic molecule towards inhibition of corrosion of steel in $0.5 \mathrm{M} \mathrm{H}_{2} \mathrm{SO}_{4}$ medium. Progress Inorganic Coatings, 51 (2),118-124 (2004).

16. Sandulescu, R., Mirel, S. and Oprean, R. The development of spectrophotometric and electroanalytical methods for ascorbic acid and acetaminophen and their applications in the analysis of effervescent dosage forms. Journal of Pharmaceutical and Biomedical Analysis 23 (1),77-87 (2000).

17. Oguzie, E. E., Wang, S. G., Li Y. and Wang, F. H. Influence of iron microstructure on corrosion inhibitor performance in acidic media. J. Phys. Chem. C, 113 (19), 84208429 (2009).

18. Issaadi, S., Douadi, T., Zouaoui, A., Chafaa, S., Khan, M.A. and Bouet, G., Novel thiophene symmetrical Schiff base compounds as corrosion inhibitor for mild steel in acidic media. Corros. Sci., 53, 1484-1488 (2011)

19. Ali, S. A., Saeed, M.T. and Rahman, S. U. The isoxazolidines: a new class of corrosion inhibitors of mild steel in acidic medium. Corros Sci., 45, 253-266(2003)

20. Yıldırım, A. and Cetin, M. Synthesis and evaluation of new long alkyl side chain acetamide, isoxazolidine and isoxazoline derivatives as corrosion inhibitors. Corros Sci, 50, 155-165 (2008).

21.Bartos, M. and Hackerman, N. A study of inhibition action of propargyl alcohol during anodic dissolution of iron in hydrochloric acid. J ElectrochemSoc 139,34283433(1992).

22.Abdel-Monem, Y. K., Amer, H. H., Mandour, H. S. and Alkafaween, I. K., Synthesis, characterization and biological activity of new metal complexes of N-(4-(2hydrazinyl-2-oxoethoxy)phenyl)acetamide. Research Journal of Pharmaceutical, Biological and Chemical Sciences 7 (3),1682-1697 (2016).

23. Stern, M. A. Method for determining corrosion rates from linear polarization data. Corrosion 14, 60-64(1958).

24. Sekunowo, O. I, Adeosun, S. O. and Lawal, G. I. Potentiostatic polarisation responses of mild steel in seawater and acid environments. International Journal of Scientific \& Technology Research 2 (10),139-145(2013).

25. Achary, G., Sachin, H. P. , Naik, Y. A. and Venkatesha, T. V. The corrosion inhibition of mild steel by 3-formyl-8-hydroxy quinoline in hydrochloric acid medium. Materials Chemistry and Physics 107,44-50(2008)

26. Li W. H., He Q., Zhang S., Pei C., and Hou B. Some new triazole derivatives as inhibitors for mild steel corrosion in acidic medium. J.Appl. Electrochem., 38,289-295 (2008).

Egypt. J. Chem. 60, No. 2 (2017) 
27. Oguzie, E. E., Inhibition of acid corrosion of mild steel by Telfaria occidentalis extract.Pigment \& Resin Technology 34, 321-326 (2005).

28. Jeyaprabha, C., Sathiyanarayanan, S. and Venkatachari, G., Corrosion inhibition of pure iron in $0.5 \mathrm{M} \mathrm{H}_{2} \mathrm{SO}_{4}$ solutions by ethanolamines. Applied Surface Science 246, 108-116 (2005)

29. Hackerman, N. and Makrids, A. C., Action of polar organic inhibitors in acid dissolution of metals. Ind. Eng. Chem., 46, 523 (1957).

30. Tezuka, M., Tomoe, Y. and Fujü, S., $7^{\text {th }}$ Int. Conf. Metall. Corros. Brazil, 1850 (1978).

31. Böhni, H., localized corrosion, in F. Mansfeld (Ed.), Corrosion Mechanism Marrcel Dekker, New York. P. 285(1987).

32. Mann, C.A., Trans Electrochem. Soc., 69,115(1936).

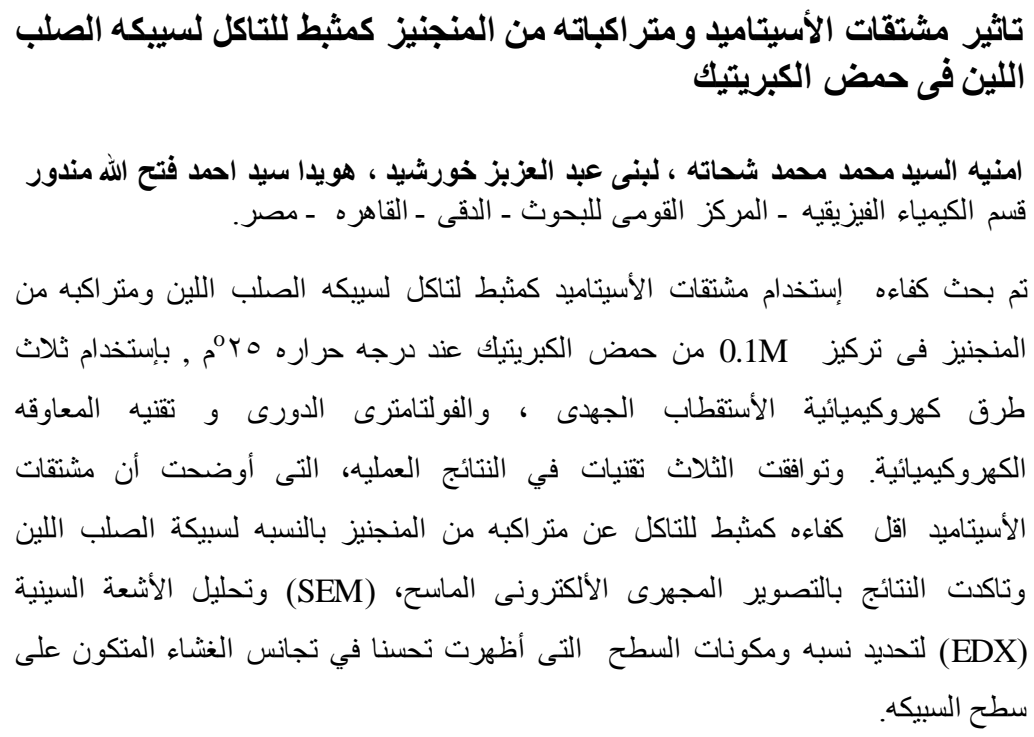

